\title{
UTILIZAÇÃO DE CRITÉRIOS METALÚRGICOS NO LINGOTAMENTO CONTÍNUO PARA CONSTRUÇÃO E VALIDAÇÃO DE UM MODELO MATEMÁTICO DA SOLIDIFICAÇÃO*
}

\author{
Francisco Diego Araruna da Silva ${ }^{1}$ \\ Alex Maia do Nascimento ${ }^{2}$ \\ Victor Alves Wanderley ${ }^{3}$ \\ Cleiton Batista Gomes ${ }^{4}$ \\ Oscar Olímpio de Araújo Filho ${ }^{5}$
}

\section{Resumo}

A necessidade de elevados patamares de competitividade na indústria do aço tem levado à utilização cada vez mais frequente, por parte daqueles que utilizam lingotamento contínuo, de ferramentas de modelagem matemática da solidificação. $A$ construção dessas ferramentas, por sua vez, possui uma etapa importante de validação, onde devem ser comparados os resultados simulados com dados reais do processo sendo estudado. Dessa forma, o presente trabalho tem como objetivo utilizar critérios metalúrgicos de qualidade e processo no lingotamento contínuo para auxiliar na construção e validação de um modelo matemático da solidificação de tarugos de aço. Uma vez que há na literatura diversas formulações para os coeficientes de transferência de calor na refrigeração primária (molde) e secundária (zona de sprays), foram realizadas análises - a luz dos critérios metalúrgicos - dos resultados simulados com nove combinações dessas formulações, sendo possível, ao final, determinar qual combinação melhor modela o processo (ou máquina) em estudo.

Palavras-chave: Lingotamento contínuo; Solidificação; Modelamento; Critérios metalúrgicos.

\section{CONSTRUCTION AND VALIDATION OF A CONTINUOUS CASTING SOLIDIFICATION MODEL USING METALLURGICAL CRITERIA}

\section{Abstract}

The need for high levels of competitiveness in the steel industry has led to increasingly use of solidification models by those who use continuous casting. During the construction of these models, it is necessary to validate the model results by comparing them with field results from the continuous casting machine being studied. Thus, this work aims to use quality and process metallurgical criteria to assist the construction and validation of a steel billet continuous casting solidification model. Once technical literature has many formulations for the heat transfer coefficients (mould and spray colling), one has analised the model results using nine combinations of these formulations and it was decided which combination resulted in the more accurate model of the process studied.

Keywords: Continuous casting; Solidification; Modeling; Metallurgical criteria.

\footnotetext{
Mestre em Engenharia de Materiais, Assessor Técnico de Aciaria, Gerdau Aços Brasil.

Doutor em Engenharia de Materiais, Consultor Técnico de P\&D, Gerdau Aços Brasil.

Graduando em Engenharia Mecânica, Universidade Federal de Pernambuco.

Graduando em Engenharia Mecânica, Faculdade Estácio, Assistente Técnico de Aciaria.

Doutor em Engenharia de Materiais, Professor Adjunto, Universidade Federal de Pernambuco.
} 


\section{INTRODUÇÃO}

O processo de lingotamento contínuo tem sido empregado em larga escala na indústria do aço no mundo inteiro [1]. A necessidade de elevados patamares de competitividade na indústria do aço tem levado à utilização cada vez mais frequente, por parte daqueles que utilizam lingotamento contínuo, de ferramentas de modelagem matemática da solidificação. O emprego dessas ferramentas permite a otimização do processo de lingotamento sem a necessidade de extensivos e custosos experimentos de campo, uma vez que é possível integrar métodos de otimização ao modelo de solidificação, robustecendo-o [2-6].

No entanto, faz-se importante uma avaliação prévia do grau de ajuste do modelo à máquina de lingotamento contínuo estudada. Isso ocorre pelo fato de que estão disponíveis na literatura diversas estratégias de modelamento e modelos de cálculo das variáveis de transferência de calor. Com o modelo devidamente validado, tem-se disponível uma ferramenta poderosa para a garantia da estabilidade operacional e para a melhoria contínua do processo.

Os perfis da temperatura da superfície, temperatura do centro e espessura solidificada, obtidos via modelamento matemático, permitem a realização de diversas avaliações acerca do processo de lingotamento contínuo simulado. Isso é possível a partir da comparação de informações extraídas dessas curvas com os critérios metalúrgicos de processo e qualidade no lingotamento contínuo disponíveis na literatura, sendo os principais:

- Critério 1: Espessura da casca solidificada na saída do molde $[3,4,7,8]$ : A espessura da casca solidificada na saída do molde deve ser maior que um valor mínimo, geralmente definido como $10 \%$ da espessura do produto final. Essa restrição implica em uma baixa probabilidade de ocorrência de breakouts causados pelas tensões de extração e a pressão ferrostática exercida pela poça líquida.

- Critério 2: Temperatura no ponto de desempeno (extração): A temperatura da superfície $\left(T_{S}\right)$ do tarugo no ponto de desempeno/extração será definido de modo evitar a zona de baixa ductilidade do aço a altas temperaturas (ou seja, evitar $\left.T>1200^{\circ} \mathrm{C}\right)[9,10]$ e a zona de baixa ductilidade do aço a baixas temperaturas (evitar $\left.\mathrm{T}<850^{\circ} \mathrm{C}\right)[9,11,12]$.

- Critério 3: Reaquecimento entre zonas [10,13]: O reaquecimento ocorre quando o tarugo passa de uma zona de refrigeração com uma maior eficiência para outra com menor taxa de resfriamento. O reaquecimento leva ao desenvolvimento de tensões trativas (causadas pela expansão da superfície) na parte interior da casca sólida a qual, como visto anteriormente, é frágil a temperaturas acima de $1200^{\circ} \mathrm{C}$ (zona de baixa ductilidade de altas temperaturas). Vistas no plano transversal, essas tensões trativas atuam paralelas à superfície, e assim essas trincas se formam perpendicularmente à superfície. Recomenda-se que o reaquecimento máximo ao longo do processo seja de $150^{\circ} \mathrm{C}$.

- Critério 4: Comprimento metalúrgico [14,15]: A solidificação do tarugo deve estar completa antes do ponto de corte do tarugo, de modo a evitar problemas de rechupe e, em casos extremos, a projeção de aço líquido ou pastoso (do núcleo do tarugo) no momento do corte.

O presente trabalho tem como objetivo utilizar critérios metalúrgicos de qualidade e processo no lingotamento contínuo para auxiliar na construção e validação de um modelo matemático da solidificação de tarugos de aço. Uma vez que há na literatura 
diversas formulações para os coeficientes de transferência de calor na refrigeração primária (molde) e secundária (zona de sprays), objetiva-se comparar os resultados simulados (usando nove combinações dessas formulações) com resultados reais do processo, visando obter a combinação de formulações que melhor modela o processo (ou máquina) em estudo.

\section{MATERIAIS E MÉTODOS}

\subsection{Modelo de Solidificação e Formulações para $h_{g}$}

O método utilizado para o modelamento da transferência de calor no lingotamento contínuo foi a mesma já adotada por diversos autores, cujas referências encontramse disponíveis na literatura [2-4,16]. No presente trabalho utilizou-se o método dos volumes finitos (MVF) para a resolução das equações de transferência de calor. As condições de contorno utilizadas nesse método de modelamento são:

- Tempo: no tempo $t=0$, o perfil de temperatura da área de controle no menisco $(z=0)$ é igual à temperatura de lingotamento;

- Linha central do tarugo: considerando um fluxo de calor simétrico nos planos centrais, o calor não flui através deles (adiabático);

- Superfície externa do tarugo: a condição de contorno da superfície representa as três zonas de resfriamento, as quais são caracterizadas pelo coeficiente de transferência de calor $h_{g}\left[\mathrm{~W} / \mathrm{m}^{2} \mathrm{~K}\right]$ de cada zona (Equação 1):

$$
\begin{array}{lll}
x=0, & 0 \leq y \leq \frac{Y}{2}, & -k \frac{\partial T}{\partial x}=h_{g}\left(T_{\text {superfície }}-T_{\text {ambiente }}\right) \\
y=0, & 0 \leq x \leq \frac{X}{2}, & -k \frac{\partial T}{\partial y}=h_{g}\left(T_{\text {superfície }}-T_{\text {ambiente }}\right)
\end{array}
$$

Para determinar ou estimar os coeficientes de transferência de calor $\left(h_{g}\right)$ metal/molde e sprays existem diversas formulações, como as apresentadas na Tabela 1 para $o$ molde e na Tabela 2 para os sprays.

\begin{tabular}{|c|c|c|}
\hline Referência/Uso & Formulação & Comentário \\
\hline $\begin{array}{l}\text { Toledo (1993) [17] } \\
\text { Tarugos e Placas }\end{array}$ & $\begin{array}{c}h_{g=}\left(0,071 \cdot e^{- \text {tempo }}+2,328 \cdot\right. \\
\left.e^{- \text {tempo } / 9,5}+0,698\right) \cdot 1000\left[\mathrm{~W} / \mathrm{m}^{2} \mathrm{~K}\right]\end{array}$ & $\begin{array}{l}\text { - Valor variável ao longo do } \\
\text { molde } \\
\text { - Obtido experimentalmente } \\
\text { onde tempo }=L_{z} / V_{\text {ling }}\end{array}$ \\
\hline $\begin{array}{l}\text { Lait (1974) [18] } \\
\text { Tarugos e Placas }\end{array}$ & $\begin{array}{c}\overline{h_{g}}=\left(1,696-0,0162 \cdot t_{\text {molde }}\right) \cdot \\
1000\left[\mathrm{~W} / \mathrm{m}^{2} \mathrm{~K}\right]\end{array}$ & $\begin{array}{l}\text { - Valor médio e constante } \\
\text { - Obtido experimentalmente }\end{array}$ \\
\hline $\begin{array}{l}\text { Silva (1996) [19] } \\
\text { Tarugos e Placas }\end{array}$ & $\begin{array}{l}\quad \overline{h_{g}}=1004,6 \cdot \exp \left(-0,02 \cdot t_{\text {molde }}\right) \\
{\left[\mathrm{W} / \mathrm{m}^{2} \mathrm{~K}\right]}\end{array}$ & $\begin{array}{l}\text { - Valor médio e constante } \\
\text { - Obtido experimentalmente }\end{array}$ \\
\hline
\end{tabular}

Tabela 1. Algumas formulações para o coeficiente de transferência de calor no molde $\left(\boldsymbol{h}_{\boldsymbol{g}}\right)$.

onde: $t_{\text {molde }}=L_{M} / V_{\text {ling }}$, tempo de residência do tarugo no molde; $L_{M}=$ comprimento do molde; $V_{\text {ling }}=$ velocidade de lingotamento $; L_{z}=$ distância percorrida em $z$. 
Tabela 2. Algumas formulações para o coeficiente de transferência de calor nos sprays.

\begin{tabular}{|c|c|c|}
\hline Referência/Uso & Formulação & $\begin{array}{l}\text { Procedimento } \\
\text { Experimental }\end{array}$ \\
\hline $\begin{array}{l}\text { Mizikar (1970) [20] } \\
\text { Tarugos e Placas }\end{array}$ & $h_{g=}[0,0776 \cdot \stackrel{o}{m}] \cdot 1000\left[\mathrm{~W} / \mathrm{m}^{2} \mathrm{~K}\right]$ & $\begin{array}{l}\text { - Placa inox } 304 \text { vertical } \\
\text { - } T_{\text {inicial }}=930^{\circ} \mathrm{C} \\
\text { - Pressão=60-620 MPa } \\
\text { - Distância } \\
\text { bicos } / \text { tarugo }=102 \mathrm{~mm}\end{array}$ \\
\hline $\begin{array}{l}\text { Nozaki (1978) [21] } \\
\text { Tarugos e Placas }\end{array}$ & $\begin{array}{c}\stackrel{o}{g}=\left[0,3925 \cdot m^{0,55} \cdot\left(1-0,0075 \cdot T_{\text {água }}\right)\right] \cdot 1000 \\
{\left[\mathrm{~W} / \mathrm{m}^{2} \mathrm{~K}\right]}\end{array}$ & $\begin{array}{l}\text { - Placa aço carbono horizontal } \\
\text { - } T_{\text {inicial }}=930^{\circ} \mathrm{C} \\
\text { - Ajuste empírico } \\
\text { - Melhor para placa }\end{array}$ \\
\hline $\begin{array}{l}\text { Bolle (1979) [22] } \\
\text { Tarugos e Placas }\end{array}$ & $h_{g}=\left[0,36 \cdot m^{0,556}\right] \cdot 1000\left[\mathrm{~W} / \mathrm{m}^{2} \mathrm{~K}\right]$ & $\begin{array}{l}\text { - Placa inox } 309 \text { horizontal } \\
\text { - } T_{\text {inicial }}=600-1100^{\circ} \mathrm{C}\end{array}$ \\
\hline
\end{tabular}

onde: $\stackrel{o}{m}=$ vazão de água [l/s]; $T_{\text {água }}=$ temperatura da água dos sprays $[\mathrm{K}]$.

Quanto ao resfriamento terciário, ou região de radiação livre, pode-se utilizar o coeficiente de transmissão de calor devido à radiação, calculado a partir da Equação 2 [23]:

$$
h_{\text {rad }}=\sigma \cdot \varepsilon \cdot\left(T_{s}+T_{a m b}\right)\left(T_{s}^{2}+T_{a m b}^{2}\right)
$$

onde $\sigma=$ constante de Stefan-Boltzman $\left(5,67 \cdot 10^{-8} \mathrm{~W} / \mathrm{m}^{2} \mathrm{~K}^{4}\right) ; \varepsilon=$ emissividade da superficie $\left(0,80\right.$ para 0 aço); $T_{s}=$ temperatura da superfície do tarugo; $T_{a m b}=$ temperatura do ambiente.

\subsection{Máquina de Lingotamento e Caracterização Macroestrutural}

A máquina de lingotamento contínuo de tarugos estudada possui dois veios e seus principais parâmetros construtivos são mostrados na Tabela 3. O aço utilizado nas simulações e corridas industriais foi o AISI 1025, cujas propriedades termofísicas podem ser encontradas na literatura [24].

Tabela 3. Dados da máquina de lingotamento estudada.

\begin{tabular}{lccl}
\hline \multicolumn{4}{c}{ Molde } \\
\hline Dimensões (lados) & $0,12 \times 0,12 \mathrm{~m}$ \\
\hline \multicolumn{4}{c}{ Sprays } \\
\hline $\begin{array}{l}\text { Comprimento da Zona 1 } \\
\text { Comprimento da Zona 2 }\end{array}$ & 0,21 & $\mathrm{~m}$ \\
\hline \multicolumn{4}{c}{ Radiação Livre } \\
\hline Distância do Menisco ao Oxicorte & 14,4 & $\mathrm{~m}$ \\
Coeficiente de Transferência de Calor & 150 & $\mathrm{~W} / \mathrm{m}^{2} \mathrm{~K}$ \\
$\left(h_{\text {rad }}\right)$ & &
\end{tabular}

Outra técnica utilizada para validação das simulações foi a macrografia de amostras das seções transversais dos tarugos. Foi realizado o lixamento com granulometrias 60 e 80 , seguido por ataque químico com solução aquosa de $\mathrm{HCl}$ a $20 \%$, de modo a revelar a presença ou não de trincas a meio caminho. 


\subsection{Seleção das Corridas Industriais}

Foram escolhidas 10 corridas industriais para a realização das avaliações dos modelos de transferência de calor no molde e zona de sprays. O critério para a escolha das corridas avaliadas foi basicamente abranger uma variada combinação de parâmetros de processo relacionados à transferência de calor, como temperatura e velocidade de lingotamento e quantidade de água na refrigeração secundária medida pela relação $\mathrm{l} / \mathrm{kg}$ (razão entre a quantidade de água total da zona de sprays e a massa de tarugo produzida). A Tabela 4 apresenta esses parâmetros. Não houveram ocorrências de breakout, defeitos superficiais (associados a temperaturas da superfície nas zonas de baixa ductilidade do aço) e rechupe (ou projeção de aço no oxicorte) nas corridas selecionadas.

Tabela 4. Corridas industriais selecionadas para análise. $T\llcorner$ e $v\llcorner$ se referem a temperatura e a velocidade de lingotamento respectivamente.

\begin{tabular}{c|cccccc} 
Corrida & $\mathrm{T}_{\mathrm{L}}\left({ }^{\circ} \mathrm{C}\right)$ & $\begin{array}{c}\mathrm{V} \mathrm{L} \\
(\mathrm{m} / \mathrm{min})\end{array}$ & $\begin{array}{c}\text { Relação } \\
\mathrm{l} / \mathrm{kg}\end{array}$ & Breakout? & $\begin{array}{c}\text { Defeitos } \\
\text { Superfi- } \\
\text { ciais? }\end{array}$ & $\begin{array}{c}\text { Rechupe } \\
\text { ou } \\
\text { projeção } \\
\text { de aço no } \\
\text { corte? }\end{array}$ \\
\hline C1 & 1557 & 2,3 & 1,5 & Não & Não & Não \\
C2 & 1538 & 2,6 & 1,1 & Não & Não & Não \\
C3 & 1544 & 2,7 & 1,5 & Não & Não & Não \\
C4 & 1556 & 2,9 & 1,4 & Não & Não & Não \\
C5 & 1547 & 2,9 & 1,6 & Não & Não & Não \\
C6 & 1578 & 2,5 & 1,2 & Não & Não & Não \\
C7 & 1551 & 2,4 & 1,2 & Não & Não & Não \\
C8 & 1553 & 2,9 & 1,5 & Não & Não & Não \\
C9 & 1540 & 2,6 & 1,3 & Não & Não & Não \\
C10 & 1525 & 2,9 & 1,1 & Não & Não & Não
\end{tabular}

Dessa forma, foram realizadas 90 simulações abrangendo todas as combinações de formulações de $h_{g}$ (três para o molde, Tabela 1, e três para os sprays, Tabela 2) das 10 condições avaliadas. A conclusão acerca de quais formulações melhor se ajustam a máquina de lingotamento contínuo estudada foi realizada comparando os resultados das simulações com os resultados reais de processo mostrados na Tabela 4.

\section{RESULTADOS E DISCUSSÃO}

\subsection{Critério Metalúrgico 1: espessura da casca sólida na saída do molde}

Como não houve ocorrência de breakout nas corridas avaliadas, pelo critério metalúrgico 1, as espessuras solidificadas na saída do molde foram maiores que $10 \%$ da espessura total do tarugo, ou seja, maiores que $12 \mathrm{~mm}$. A Tabela 5 mostra os resultados de espessura na saída do molde para todas as combinações avaliadas. Observa-se que Silva (1994) modelou uma menor transferência de calor no molde, acarretando em espessuras reduzidas da casca sólida ao final da refrigeração primária. A formulação de Lait (1974), por sua vez, foi capaz de identificar três condições críticas para a ocorrência de breakout, pelo emprego de altas velocidades 
de lingotamento em maiores superaquecimentos (diferença entre a temperatura no distribuidor e a temperatura liquidus do aço). Já as com Toledo (1993) não detectou nenhuma condição de alerta em relação a breakout.

Tabela 5. Espessuras na saída do molde segundo as simulações. Células em vermelho indicam não atendimento ao Critério 1.

\begin{tabular}{r|ccc} 
& Toledo (1993) & Lait (1974) & Silva (1996) \\
\hline C1 & 15,5 & 13,5 & 9,6 \\
C2 & 14,9 & 12,8 & 9,4 \\
C3 & 14,2 & 12,5 & 9,0 \\
C4 & 14,0 & 11,8 & 8,8 \\
C5 & 13,7 & 11,8 & 8,4 \\
C6 & 14,4 & 12,7 & 8,9 \\
C7 & 15,4 & 13,3 & 9,6 \\
C8 & 13,8 & 11,8 & 8,5 \\
C9 & 14,8 & 12,8 & 9,3 \\
C10 & 14,1 & 12,1 & 9,0
\end{tabular}

De modo a realizar uma melhor avaliação dos modelos quanto a capacidade de previsão de breakout, foi analisada uma corrida extra na qual houve a ocorrência de um breakout devido um elevado superaquecimento $\left(71^{\circ} \mathrm{C}\right)$. A Figura 1 mostra 0 detalhe dos perfis de espessura solidificada próximo à saída do molde, destacando a região de risco de breakout. Observa-se que a simulação com Toledo (1993) não foi capaz de detectar o risco da ocorrência do breakout que realmente ocorreu nesse caso.

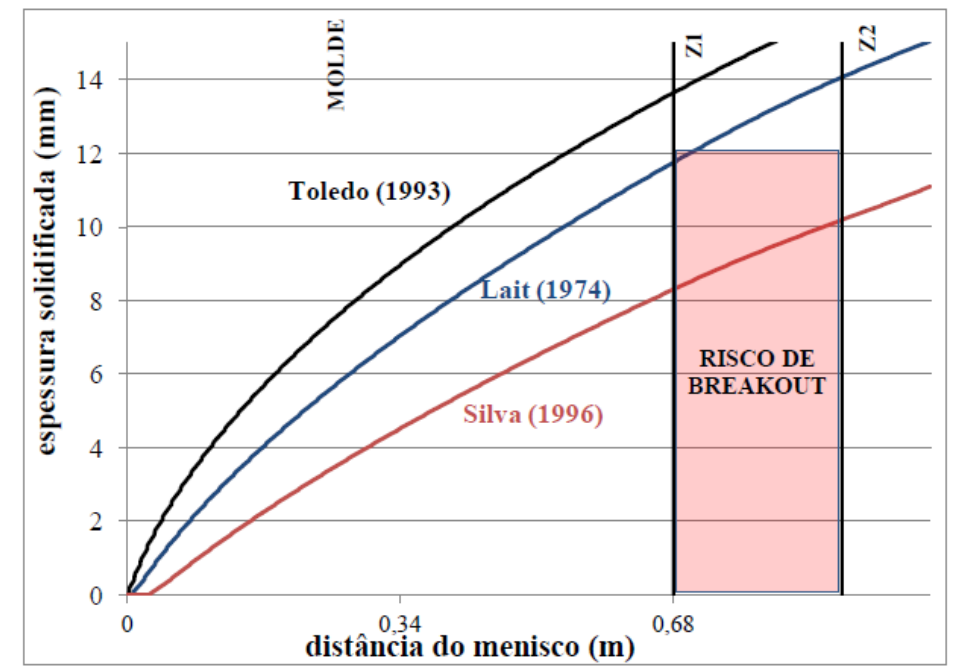

Figura 1. Curva de espessura da casca sólida da corrida em que houve breakout, detalhando somente a região do molde e início da zona de sprays.

\subsection{Critério Metalúrgico 2: temperatura da superfície no desempeno}

Todas as combinações de formulações do hg do molde e sprays possuíram boa assertividade em relação ao critério metalúrgico 2, uma vez que foram capazes de mostrar que nenhuma corrida apresentou temperaturas dentro das zonas de baixa ductilidade do aço - o que de fato ocorreu na realidade uma vez que não foram observados defeitos superficiais associados a temperaturas fora da faixa ótima no ponto de desempeno/extração. A Figura 2 mostra o exemplo das simulações da 
temperatura da superfície das 10 corridas utilizando Lait (1974) + Nozaki (1978), indicando que nenhuma condição apresenta temperaturas fora da faixa ótima $\left(850^{\circ} \mathrm{C}<\mathrm{T}<1200^{\circ} \mathrm{C}\right)$ no desempeno.

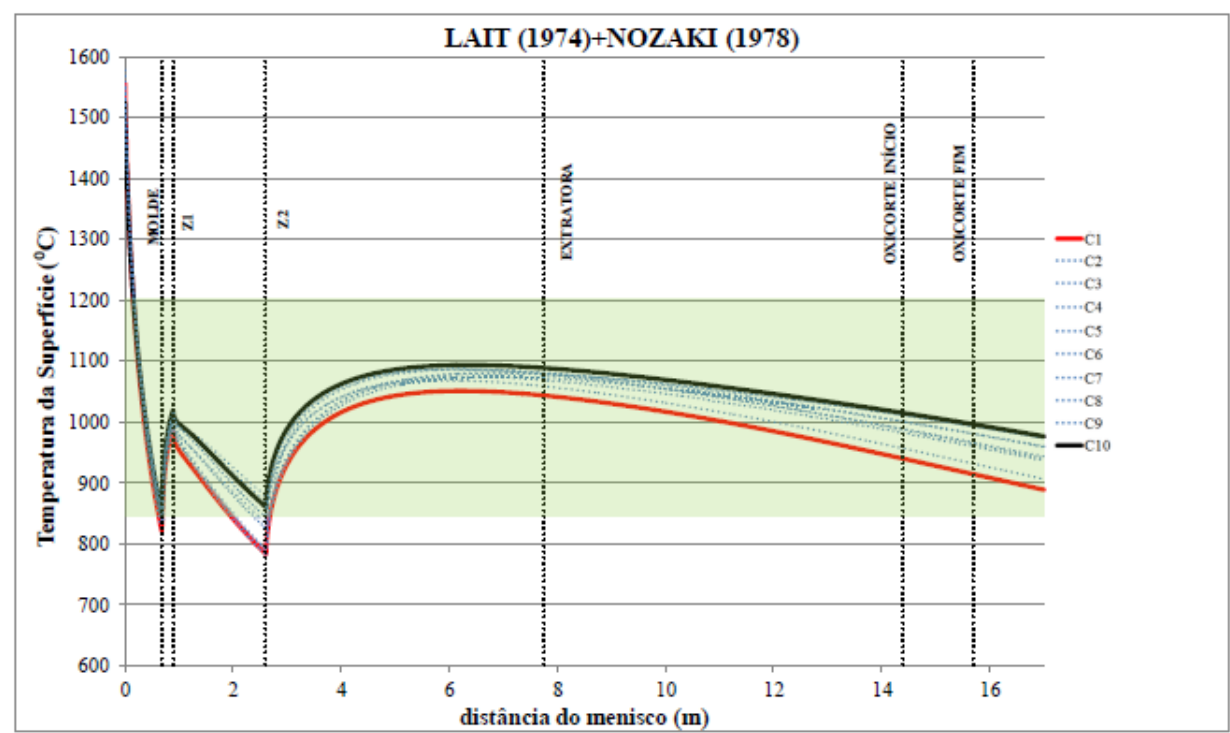

Figura 2. Simulações da temperatura da superfície do tarugo de todas as corridas industriais, sombreando a região de atendimento ao Critério 2.

\subsection{Critério Metalúrgico 3: reaquecimento entre zonas}

Somente as combinações Toledo (1993) + Nozaki (1978) e Lait (1974) + Nozaki (1978) foram capazes de prever corretamente todas as ocorrências de trincas a meio caminho observadas nas macrografias - isto é, resultaram em simulações que indicaram reaquecimentos acima de $150^{\circ} \mathrm{C}$ nos pontos em que foram encontradas trincas a meio caminho.

A Figura 3a mostra um exemplo da análise da corrida C10 a luz do Critério Metalúrgico 3. Nela são mostradas as curvas de temperatura da superfície ("Ts"), temperatura do centro ("Tc") e espessura solidificada ("e", no eixo secundário). Observa-se dois pontos em que houve reaquecimento acima de 150두 : o primeiro na saída do molde - em que há uma espessura solidificada em torno de $12 \mathrm{~mm}$ - e o segundo após a Z2 (entrada da zona de radiação livre) - em que há a uma espessura sólida em torno de $30 \mathrm{~mm}$. De fato, foram observadas trincas a meio caminho nesses pontos sinalizados como críticos na simulação, conforme mostra a macrografia de um tarugo dessa corrida na Figura 3b. As trincas de número 1 e 2 podem ser associadas ao reaquecimento na saída do molde, e a trinca 3 ao reaquecimento na zona de radiação livre. 


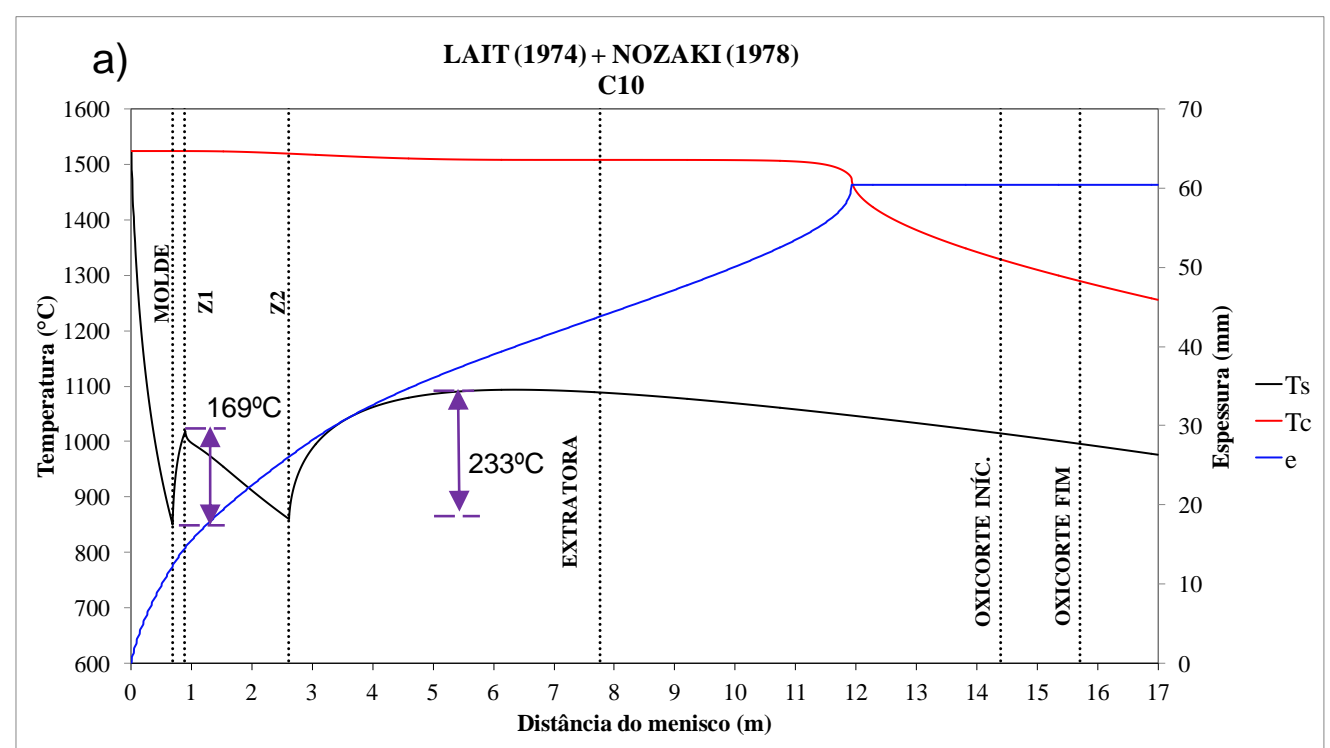

b)

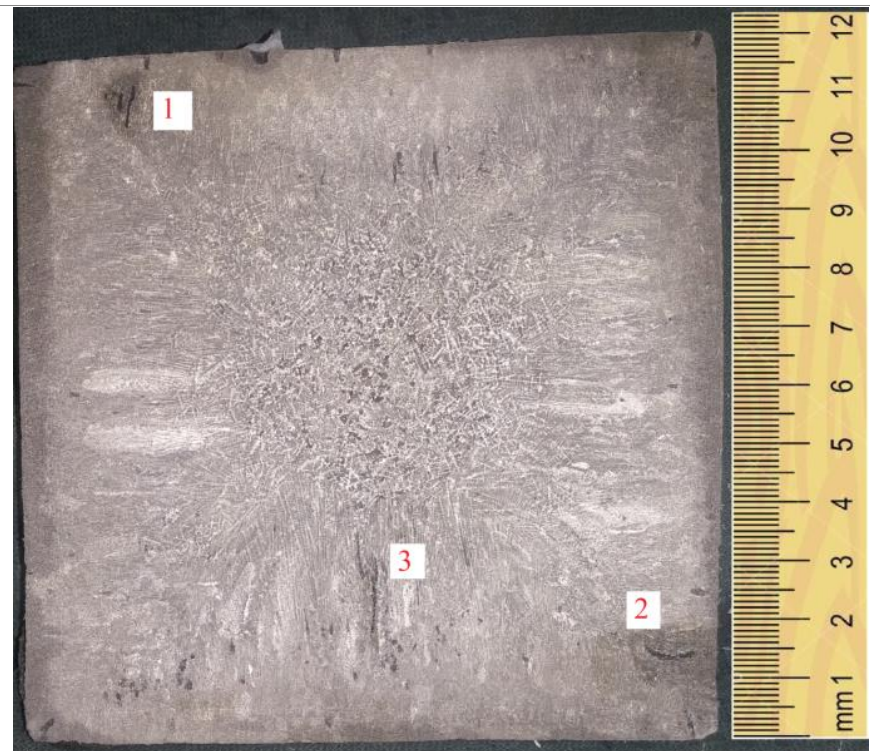

Figura 3. Curvas simuladas da condição C10 com a combinação Lait (1974) + Nozaki (1978) e b) macrografia de uma amostra de $\mathrm{C} 10$, destacando trincas.

\subsection{Critério Metalúrgico 4: fechamento da espessura}

Como em nenhuma das corridas estudadas foi observada a presença de rechupe severo ou projeção de aço no corte, o comprimento metalúrgico real em todas as corridas foi menor que $14,4 \mathrm{~m}$ (posição do oxicorte na máquina em estudo). Somente a combinação Silva (1996) + Mizikar (1970) indicou comprimento metalúrgico acima de $14,4 \mathrm{~m}$.

\subsection{Resumo das avaliações}

Após todas as avaliações dos modelos, pode-se construir o resumo mostrado na Tabela 6 . As células sombreadas mostram os critérios atendidos de forma satisfatória pela combinação de modelos em questão.

Conclui-se assim, que a máquina de lingotamento contínuo estudada é melhor modelada utilizando o modelo de Lait (1974) para o cálculo do $h_{g}$ do molde e o de Nozaki (1978) para o cálculo do $h_{g}$ da zona de sprays. É importante salientar que se 
faz necessário um estudo de cada máquina a ser modelada, de modo a garantir um modelo confiável para análises e otimização do processo.

Tabela 6. Resumo das avaliações dos modelos.

\begin{tabular}{|r|r|r|r|c|c|}
\cline { 2 - 5 } \multicolumn{1}{c|}{} & $\begin{array}{c}\text { Critério } \\
\text { Metal. 1: } \\
\text { espessura } \\
\text { molde }\end{array}$ & $\begin{array}{c}\text { Critério } \\
\text { Metal. 2: } \\
\text { temperatura } \\
\text { no desem- } \\
\text { peno }\end{array}$ & $\begin{array}{c}\text { Critério } \\
\text { Metal. 3: } \\
\text { reaque- } \\
\text { cimento } \\
\text { entre zonas }\end{array}$ & $\begin{array}{c}\text { Critério } \\
\text { Metal. 4: } \\
\text { compri- } \\
\text { mento } \\
\text { metalúrgico }\end{array}$ & $\begin{array}{c}\text { Número de } \\
\text { critérios } \\
\text { atendidos }\end{array}$ \\
\hline TOLEDO(1993)+BOLLE(1979) & & & & & 2 \\
\hline TOLEDO(1993)+NOZAKI(1978) & & & & & 3 \\
\hline TOLEDO(1993)+MIZIKAR(1970) & & & & \\
\hline LAIT(1974)+BOLLE(1979) & & & & \\
\hline LAIT(1974)+NOZAKI(1978) & & & & & 2 \\
\hline LAIT(1974)+MIZIKAR(1970) & & & & & 2 \\
\hline SILVA(1996)+BOLLE(1979) & & & & & 2 \\
\hline SILVA(1996)+NOZAKI(1978) & & & & & 2 \\
\hline SILVA(1996)+MIZIKAR(1970) & & & & & 2 \\
\hline
\end{tabular}

Ao modelo final validado foi integrado um algoritmo de otimização da refrigeração secundária, de modo a obter a curva otimizada de solidificação (visão qualidade e custos) no lingotamento contínuo. Tem-se assim, uma ferramenta robusta para a melhoria contínua do processo. Detalhes sobre a otimização serão publicados em trabalhos posteriores.

\section{CONCLUSÃO}

O modelo matemático de transferência de calor no lingotamento contínuo, construído a partir dos modelos de Lait (1974) e Nozaki (1978) para os coeficientes de transferência de calor no molde e zona de sprays respectivamente, apresentou um excelente grau de ajuste aos resultados reais obtidos da máquina de lingotamento estudada.

É importante salientar que se faz necessário um estudo de cada máquina a ser estudada, de modo a garantir um modelo confiável para análises e otimização do processo.

\section{Agradecimentos}

Os autores gostariam de agradecer a Gerdau por fornecer os recursos tecnológicos e humanos para a realização desse trabalho, bem como ao Programa de PósGraduação do Departamento de Engenharia Mecânica da Universidade Federal de Pernambuco.

\section{REFERÊNCIAS}

1 Won, Y.M., et al. Analysis of Solidification Cracking Using the Specific Crack Susceptibility. ISIJ International, 2000, Vol. 40, 2, pp. 129-136.

2 Silva, F.D.A. da, Otimização da Qualidade no Lingotamento Contínuo de Tarugos Através da Utilização de Modelos Matemáticos. 2015, Dissertação de Mestrado, UFPE. 
3 Cheung, N., et al. Application of a heuristic search technique for the improvement of spray zones cooling conditions in continuously cast steel billets. Applied Mathematical Modelling. 2006, Vol. 30, pp. 104-115.

4 Santos, C.A, Spim, J.A. e Garcia, A. Mathematical modeling and optimization strategies (genetic algorithm and knowledge base) applied to the continuous casting of steel. Engineering Applications of Artificial Intelligence. 2003, 16, pp. 511-527.

5 Feng, H., et al. Generalized constructal optimization for solidification heat transfer process of slab continuous casting based on heat loss rate. Energy. 2014, Vol. 66, pp. 991-998.

6 Klimes, L. e Stetina, J. A rapid GPU-based heat transfer and solidification model for dynamic computer simulations of continuous steel casting. Journal of Materials Processing Technology. December 2015, 2015, Vol. 226, pp. 1-14.

7 Brimacombe, J.K. Design of continuous casting machines based on heatflow analysis: state-of-the art review. Continuous Casting - Heat Flow, Solidification and Crack Formation. 1984, Vol. 2, pp. 17-28.

8 Lait, J.E. e Brimacombe, J.K. Solidification During Continuous Casting of Steel. Transactions of the Iron and Steel Society of AIME. 1982, 1, pp. 1-13.

9 Thomas, B.G., Brimacombe, J.K e Samarasekera, I.V. The Formation of Panel Cracks in Steel Ingots: A State-of-the-Art Review. ISS Transactions. 1986, Vol. 7, pp. 7-20.

10 Brimacombe, J.K. e Sorimachi, K. Crack Formation in the Continuous Casting of Steel. Metallurgical Transactions B. 1977, Vol. 8, n. 3, pp. 489-505.

11 Ericson, L. Cracking in Low Alloy Aluminum Grain Refined Steels. Scand. J. Metallurgy. 1977, Vol. 6, n. 3, pp. 116-124.

12 Suzuki, H.G., et al. Hot Ductility in Steels in the Temperature Range Between 900 and $600^{\circ} \mathrm{C}$. Tetsu-to-Hagane. 1981, Vol. 67, n. 8, pp. 1180-1189.

13 Brimacombe, J.K., Samarasekera, I.V. e Lait, J.E. Continuous Casting - Heat Flow, Solidification and Crack Formation. 1984, Iron \& Steel Society of AIME.

14 Kulkarni, M.S. e Subash Babu, A. Managing quality in continuous casting process using product quality model and simulated annealing. Journal of Materials Processing Technology. 2005, 166, pp. 294-306.

15 Spuy, D., et al. An optimization procedure for the secondary cooling zone of a continuous billet caster. 1999, The Journal of The South African Institute of Mining and Metallurgy.

16 Garcia, A., et al. Lingotamento Contínuo de Aços. São Paulo : ABM, 2006. ISBN 857737-005-4.

17 Toledo, G.A., Lainez, J. e Cirión, J.C. Model Optimization of Continuous Casting Steel Secondary Cooling. Materials Science and Engineering A. 1993, Vol. 173, pp. 287-291.

18 Lait, J.E., Brimacombe, J.K. e Weinberg, F. Mathematical Modelling of Heat Flow in the Continuous Casting of Steel. s.I. : Ironmaking and Steelmaking (Quaterly), 1974. pp. 9097. Vol. 2.

19 Silva, S.P.S. Simulação matemática da influência da composição de carbono do aço na previsão de defeitos no lingotamento contínuo. 1996, Dissertação apresentada à EEIMVR-UUF. Volta Redonda : UUF.

20 Mizikar, E.A. Spray Cooling Investigation for Continuous Casting of Billets and Blooms. Iron and Steel Engineering. 1970, pp. 53-60.

21 Nozaki, T. et al. A Secondary Cooling Patern for Preventing Surface Cracks of Continuous Casting Slabs. 1978, Transactions ISIJ, Vol. 18, pp. 330-338.

22 Bolle, E. e Moreau, J.C. Spray Cooling of Hot Surfaces: a Description of the Dispersed Phase and a Parametric Study of Heat Transfer Results. 1979, Proceedings of Two Phase Flows and Heat Transfer. NATO Advanced Study Institute, Vol. III, pp. 13271346.

23 Garcia, A. Transferência de Calor e Solidificação no Processo de Lingotamento Contínuo. 1981, Tese de Livre Docência apresentada à Universidade Estadual de Campinas. Campinas : UNICAMP.

24 Toloukian, Y.S. Thermophysical Properties of Matter. New Yortk : IFI/Plenum, 1970. 